\title{
Crystallographic characterizations of materials for clean energies applications
}

\author{
Adriana Serquis \\ Centro Atómico Bariloche CNEA
}

\begin{abstract}
Materials play a key role in the search for solutions to energy problems. One attractive solution for the transition period between fossil fuels and hydrogen is the development of materials for electrochemical devices such as Solid Oxide Cells (SOC). SOC are highly efficient electrochemical devices which can convert a wide range of fuels (i.e. hydrogen, methane, carbon monoxide) into electrical energy and heat in fuel mode (SOFC) or can generate hydrogen in electroliser mode (SOEC).

In this talk I will present some of the latest results obtained in our group involving several ceramic materials that are intended for SOC devices. The focus will be placed on the correlation between the microstructural aspects (determined by the composition and processing parameters) and their physical properties that determine the materials efficiency. The structural stability and chemical compatibility between the electrode and electrolyte materials studied were evaluated through the combination of several characterization techniques, such as electron microscopies (SEM, TEM), X-ray diffraction (XRD), chemical analysis by EDS and synchrotron radiation methods (XANES y EXAFS), including some in-situ and in-operando techniques.
\end{abstract}

